\title{
Digital Impressions and Immediate Chairside Zirconia Crowns in Paediatric Dentistry: A Case Report
}

\author{
Gaurav Gupta ${ }^{1 *}$, Gupta RK ${ }^{1}$, Priyanka Gupta ${ }^{2}$ and Neelja Gupta ${ }^{1}$ \\ ${ }^{1}$ Private Practice, Wisdom Dental Clinics, India \\ ${ }^{2} S R$ Demonstrator, RUHS College of Dental Sciences, India
}

*Corresponding author: Gaurav Gupta, Private Practice, Wisdom Dental Clinics, India

\begin{abstract}
Early Childhood Caries (ECC) is a public health problem of global proportions, which involves the early carious involvement of the primary maxillary incisors followed by the maxillary and mandibular first primary molars and the mandibular cuspids. For many years, the preformed paediatric metal crown (stainless steel crown (SSC)) was considered as a gold standard. However, to over disadvantages of SSC preformed pediatric zirconia crowns are introduced recently. Some drawbacks which limit the use of zirconia crowns are that it requires significantly more time to prepare the tooth for fitting the crown and its inability to manipulate its margin and adaptability. To overcome disadvantages of Preformed Zirconia Crowns Computer-aided design and manufacturing (CAD/CAM) technology came to rescue and made enormous improvements since its introduction. In this article, through a clinical case, we present the protocol and implementation of a paediatric zirconia crown prepared with digital workflow and its followup. the restoration performance is excellent exhibiting no chipping, no discoloration, and displays an excellent marginal fit and no plaque deposition.
\end{abstract}

Keywords: CAD/CAM; digital workflow; paediatric zirconia crowns; stainless steel crown

Abbreviations: ECC: Early Childhood Caries; SSC: Stainless Steel Crown; CAD: Computer-Aided Design; CAM: Computer-Aided Manufacturing; PMs: Primary Molars; NOIS: Nitrous Oxide Inhalational Sedation

\section{Introduction}

Early Childhood Caries (ECC) is a public health problem of global proportions, which involves the early carious involvement of the primary maxillary incisors followed by the maxillary and mandibular first primary molars and the mandibular cuspids [1]. Aesthetic restoration of these carious or traumatised primary anterior teeth pose big challenge especially due to their smaller dimensions, close proximity of pulp chamber, comparatively thin enamel leading to lesser surface area for bonding as well as issues related to child behaviour and cost of treatment [2]. For many years, the preformed paediatric metal crown (stainless steel crown (SSC)) was considered as a gold standard and the best method of restoring primary molars (PMs) affected by severe carious lesions because of its higher longevity than conventional restorations using various restorative materials like silver amalgam, composites and glass ionomer cements [3]. Guidelines in paediatric restorative dentistry recommend the use of preformed paediatric stainless-steel crowns (SSCs) in cases of severe tooth decay of at least two surfaces. This clinically effective and safe restorative option is frequently refused by parents for aesthetic reasons; they prefer conventional restorations using aesthetic filling materials (composites, glass ionomer) if lesion severity limited to two surfaces permits [4]. Some drawbacks which limit the use of zirconia crowns are that it requires significantly more time to prepare the tooth for fitting the crown. Bleeding from the gum, due to anxiety or inflammation, may hinder the setting of the cement used to bond the zirconia crown to the tooth. With crying or inability to sit still and fully cooperate for the procedure, an SSC would be preferable; since the preparation of the tooth and fitting an SSC takes much less time, but with the latest innovation's manufacturers are trying to minimize these factors (Figure 1). To overcome disadvantages of Preformed 
Zirconia Crowns Computer-aided design and manufacturing (CAD/CAM) technology came to rescue and made enormous improvements since its introduction by Dr Francois Duret8 and Dr Werner Mormann.9 Nowadays, this technology is available directly in dental clinics and it is capable, via its software, of fabricating (customized) full ceramic crowns, inlays, onlays, and veneers for permanent dentition at one appointment. The materials that are used in CAD/CAM include ceramic, resin ceramic, hybrid ceramic, and zirconia blocks (Figure 2). The superior mechanical properties of these materials support the use of CAD/CAM as a trustworthy method for dental patients because it results in a high survival rate of the restorations with a low rate of restoration fracture and longterm clinical survivability [5-7]. In this article, through a clinical case, we present the protocol and implementation of a paediatric zirconia crown prepared with digital workflow and its follow-up (Figure 3).

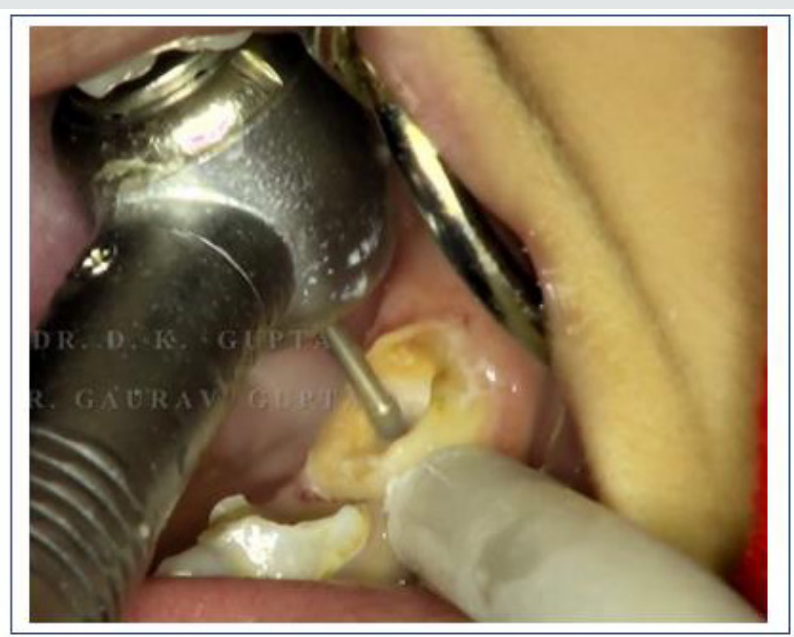

Figure 1: Access Cavity Preparation.

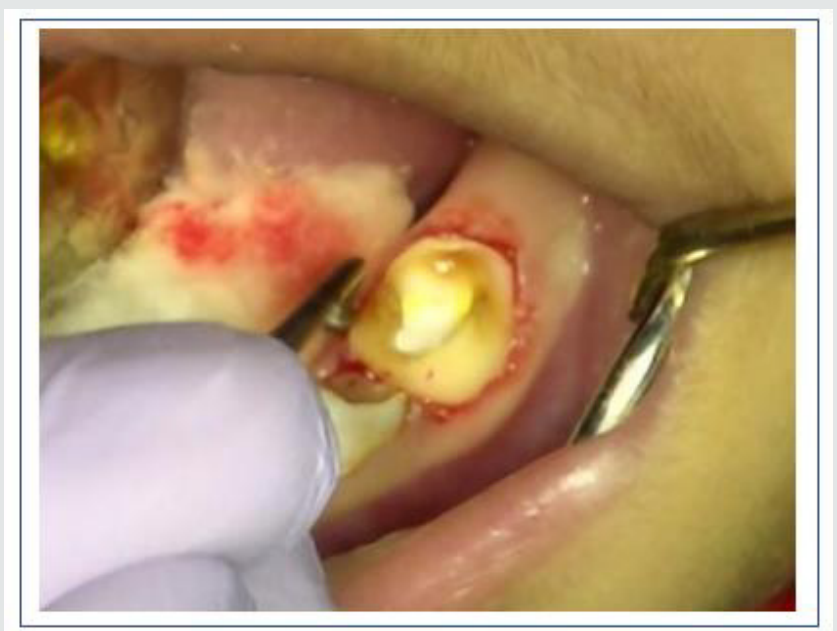

Figure 2: Pulpectomy and Prepared Tooth.

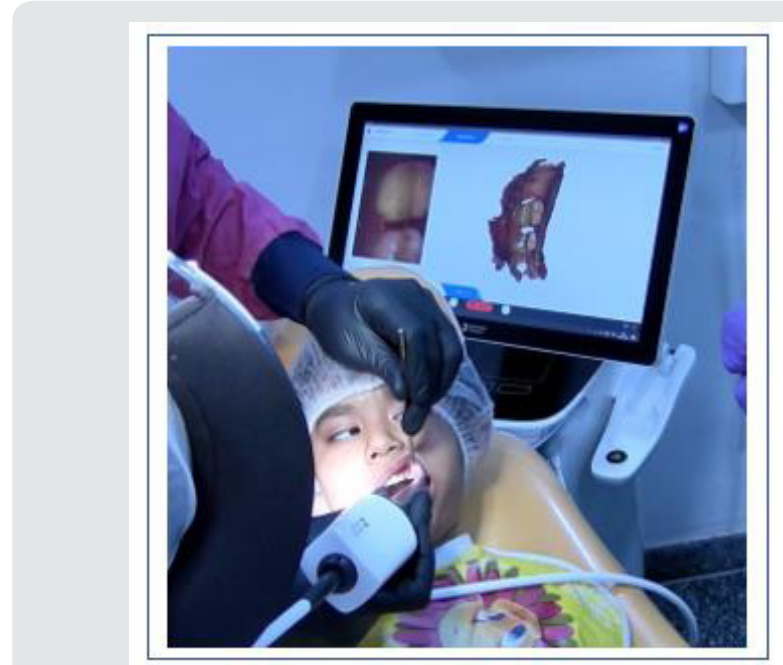

Figure 3: Digital Impression Making.

\section{Case Report}

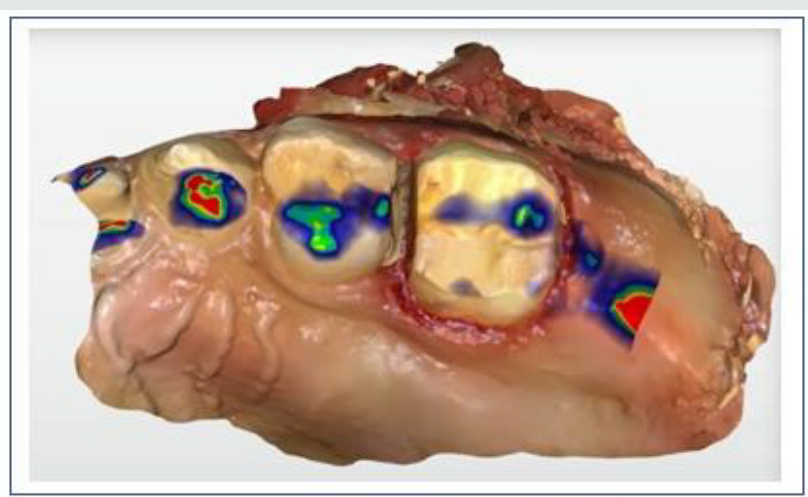

Figure 4: Final Digital Impression.

This paper describes the restoration of a primary mandibular right first molar in an 8-year-old boy. The child presented with a high risk of caries. The tooth required a full-coverage restoration, and both the child and the parent were highly concerned with the aesthetic appearance of the restoration. A medical history was taken, followed by a clinical and radiographic examination which revealed deep dentinal caries on the tooth in question tooth without any interradicular lesion. We decided to restore the primary mandibular right first molar with a paediatric zirconia crown using intraoral scanning and chairside milling (Figure 4). We have modified our protocol for the procedure exclusively for digital dentistry in paediatric patients. After diagnosis and planning we achieve local anesthesia by with or without NOIS (Nitrous Oxide Inhalational Sedation) whichever is suitable as per patients' condition. Then we directly go for tooth preparation followed by digital impression using intraoral scanner (Dentsply Omnicam). After this we perform Pulpectomy procedure meanwhile designing 
and milling of the restoration was done by another operator (Figure 5). As pulpectomy is undergoing we prepare prosthesis with chairside milling after that sintering was done which provide adequate strength and glazing is done prior to cementation which makes surface more plaque resistant. The tooth and the crown were cleaned of all blood residues. Haemostasis of the gingiva was obtained via pressure applied with a finger. A glass ionomer cement (Fuji One PLUS, GC, Louvain, Belgium) was used for the cementation (Figure 6).

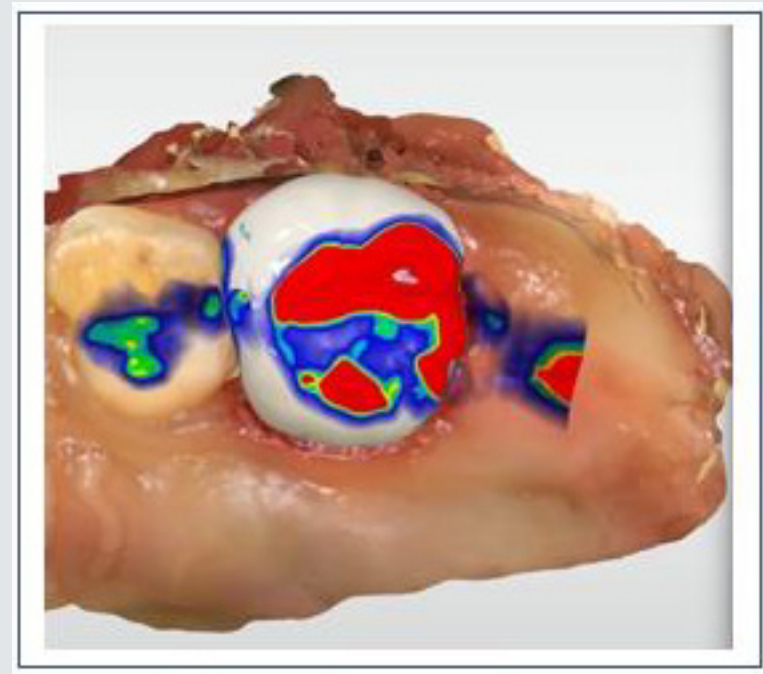

Figure 5: Final Digital Crown Designing.

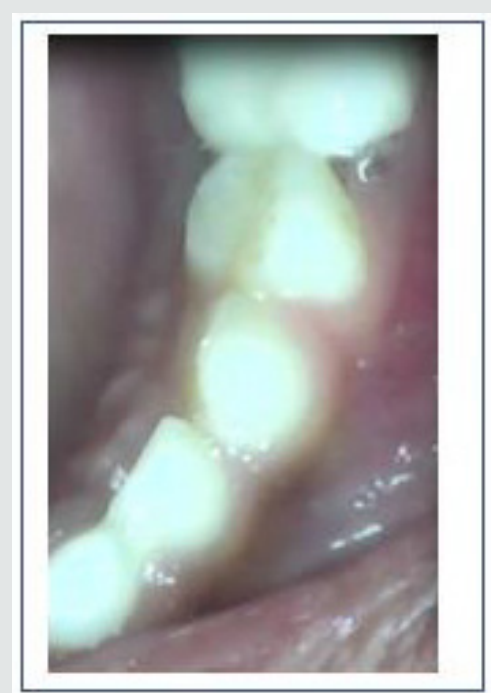

Figure 6: Delivered Prosthesis.

\section{Intraoral scanning and milling}

Quarters arch scans were made using a powder-free intraoral scanning device (Cerec AC, Omnicam, Sirona Dental Systems GmbH, Bensheim, Germany). The design mode of the restoration was completed using the Biogeneric Copy. The software optical images included, as stated: the "upper jaw," the "lower jaw," the "buccal," and the "biocopy upper." In the next step, the software automatically aligns the upper and lower jaws and articulates the models in the maximum intercuspal position. The gingival margins were defined automatically and manually designed using the "draw margin" tool. Cerec SW 4.6 automatically calculates the insertion axis and also provides tools to adjust the restoration design, including the occlusal and interproximal occlusal contact points. In the milling preview, the restoration is placed in a hybrid ceramic block (Vita Enamic, H Rauter GmbH \& Co. KG, Germany) with a shade of 2M2HT and EM-10 size that was automatically determined by the software with the shade analysis tool. The milling of the block was completed in the "Standard" mode and milled with the CEREC PRIMEMILL milling unit and diamond burs. After the completion of the milling procedure, the crown is glazed with speed fire according to the specifications from the manufacturer. The restoration was cleaned with alcohol and dried with oil- and water-free air. The inner surface of the crown is etched with $5 \%$ hydrofluoric acid for 60 seconds and then placed in an ultrasonic bath for 5 minutes. The editing time of the restoration had a duration of 2 minutes, the milling time took 9 minutes, while the total chairside time was 50 minutes.

\section{Discussion}

Primary incisors have enjoyed less retention of intracoronal restorations with tooth-coloured materials such as composite, compomer and conventional or resin modified glass ionomer due to morphology of the pulp, dentin and enamel as reported by Waggoner [1994], Piyapinyo and White [1998] and Kopel \& Beaver [1967] [8]. At present, the success and reliability of SSCs is known. Even if SCCs are recommended in the treatment of severe tooth decay in children, few dental practitioners adopt their use in clinical practice; one of the reasons for this is their poor aesthetic appearance. An aesthetic alternative to SSCs is Zirconia Crowns $[9,10]$. To date, only a few case series focusing on restoration of PMs with ZCs have been published; these concluded that they perform well [11]. It is difficult to adjust a zirconia crown because it is ceramic and cannot be trimmed. with scissors like a traditional SSC, it is necessary to use a high speed, fine diamond burs with lots of water because excessive heat could cause fractures in the crown's ceramic structure. Occlusal and interproximal adjustments are not recommended, as these will remove the crown's glaze and possibly create a weak area of thin ceramic. It is very important that zirconia crowns fit passively because they are made of solid zirconia and do not flex, attempt to sit with force will result in fracture and adjustment with bur result in microfracture. The appropriate size crown should fit passively and completely sub-gingivally without distorting the gingival tissue. All later described disadvantages of preformed zirconia crowns are successfully rectified with chair side CAD/CAM. This case report describes a single-visit fabrication of a hybrid ceramic crown on a primary molar. To limit chairside 
time and promote the quality of care given, CAD/CAM technology could be beneficially used in cases when a crown on primary tooth CAD/CAM technology is an innovative method of producing indirect restorations in primary teeth. With the help of the design software, the clinician can produce ideal occlusal and proximal contact points and a better marginal fit at the gingival wall. Moreover, it involves a shorter clinical working time, less wear of the opposing dentition, and the choice of more biocompatible materials. To the best of our knowledge, this is the first case report of a CAD/CAM crown on a first primary molar in the literature. However, there are limited cases of restorations on primary teeth with CAD/CAM technology that are associated with the fabrication of inlays or onlays for a primary decayed tooth or the fabrication of an end crown. To conclude the restoration performance is excellent exhibiting no chipping, no discoloration, and displays an excellent marginal fit and no plaque deposition. The preventive nature of glazed prosthesis showed better plaque resistance than adjacent natural tooth. The patient was placed under a prevention treatment plan of high-risk caries.

\section{References}

1. Wayne A, Darwish S, Adenubi J, Battata S, Khan N (2001) The prevalence and pattern of nursing caries in Saudi preschool children. Int J Pediat Dent 11: 361-364.
2. Donly K (2002) Pediatric Restorative Dentistry. Consensus Conference. April 15-16, 2002. San Antonio, Texas. Pediatr Dent 24: 374-376.

3. Innes NPT, Ricketts DNJ, Evans DJP (2007) Preformed metal crowns for decayed primary molar teeth. Cochrane Database Syst Rev 1: CD005512.

4. Lopez-Cazaux S, Aiem E, Velly AM, Muller-Bolla M (2019) Preformed paediatric zirconia crown versus preformed paediatric metal crown: study protocol for a randomized clinical trial. Trials 20(1): 530.

5. Mormann WH (2006) The evolution of the CEREC system. JADA 137(Suppl:): 7-13.

6. Collares K, Correa MB (2016) A practice-based research network on the survival of ceramic inlay/onlay restorations. Dent Mater 32(5): 687-694.

7. Mourouzis P, Arhakis A, Tolidis K (2019) Computer-aided Design and Manufacturing Crown on Primary Molars: An Innovative Case Report. Int J Clin Pediatr Dent 12(1): 76-79.

8. Walia T, Salami AA, Bashiri R, Hamoodi OM, Rashid F (2014) A randomised controlled trial of three aesthetic full-coronal restorations in primary maxillary teeth. Eur J Paediatr Dent 15(2): 113-118.

9. Maciel R, Salvador D, Azoubel K, Redivivo R, Maciel C, et al. (2017) The opinion of children and their parents about four different types of dental restorations in a public health service in Brazil. Eur Arch Paediatr Dent 18(1): 25-29.

10. Karaca S, Ozbay G, Kargul B (2013) Primary zirconia crown restorations for children with early childhood caries. Acta Stomatol Croat 47: 64-71.

11. Soxman JA (2015) The Handbook of Clinical Techniques in Pediatric Dentistry. Hoboken, Wiley-Blackwell, USA p. 47-50.

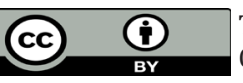

This work is licensed under Creative Commons Attribution 4.0 License

To Submit Your Article Click Here: Submit Article

DOI: $10.32474 /$ IPDOAJ.2021.05.000216

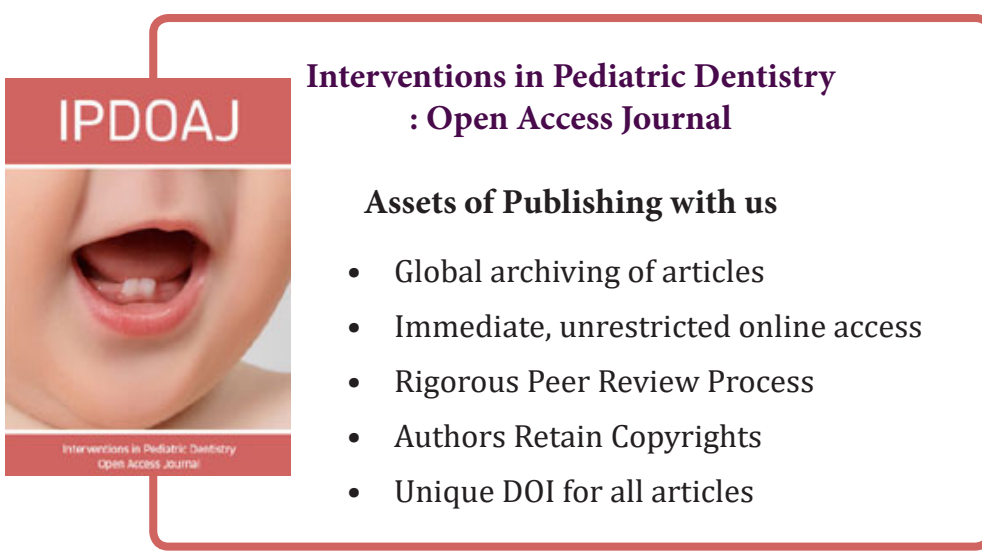

